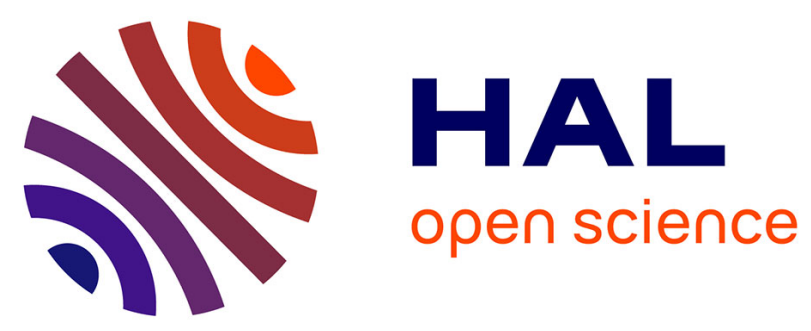

\title{
L'URBANISATION RURALE EN CHINE: TRANSFORMATIONS SOCIALES ET EFFETS D'AUBAINE ÉCONOMIQUES. LE CAS DE LA CITÉ UNIVERSITAIRE DAXUECHENG DANS LA PÉRIPHÉRIE DE LA MÉTROPOLE CANTONAISE
}

Christophe Guibert, Benjamin Taunay

\section{To cite this version:}

Christophe Guibert, Benjamin Taunay. L'URBANISATION RURALE EN CHINE: TRANSFORMATIONS SOCIALES ET EFFETS D'AUBAINE ÉCONOMIQUES. LE CAS DE LA CITÉ UNIVERSITAIRE DAXUECHENG DANS LA PÉRIPHÉRIE DE LA MÉTROPOLE CANTONAISE. Autrepart - Revue de sciences sociales au Sud, 2018. halshs-03500836

\section{HAL Id: halshs-03500836 \\ https://shs.hal.science/halshs-03500836}

Submitted on 22 Dec 2021

HAL is a multi-disciplinary open access archive for the deposit and dissemination of scientific research documents, whether they are published or not. The documents may come from teaching and research institutions in France or abroad, or from public or private research centers.
L'archive ouverte pluridisciplinaire HAL, est destinée au dépôt et à la diffusion de documents scientifiques de niveau recherche, publiés ou non, émanant des établissements d'enseignement et de recherche français ou étrangers, des laboratoires publics ou privés. 


\section{L'URBANISATION RURALE EN CHINE : TRANSFORMATIONS SOCIALES ET EFFETS D'AUBAINE ÉCONOMIQUES. LE CAS DE LA CITÉ UNIVERSITAIRE DAXUECHENG DANS LA PÉRIPHÉRIE DE LA MÉTROPOLE CANTONAISE}

\section{Christophe Guibert, Benjamin Taunay}

Presses de Sciences Po | «Autrepart »

$2018 / 2 \mathrm{~N}^{\circ} 86$ | pages 79 à 102

ISSN 1278-3986

ISBN 9782724636222

Article disponible en ligne à l'adresse :

https://www.cairn.info/revue-autrepart-2018-2-page-79.htm

Distribution électronique Cairn.info pour Presses de Sciences Po.

(C) Presses de Sciences Po. Tous droits réservés pour tous pays.

La reproduction ou représentation de cet article, notamment par photocopie, n'est autorisée que dans les limites des conditions générales d'utilisation du site ou, le cas échéant, des conditions générales de la licence souscrite par votre établissement. Toute autre reproduction ou représentation, en tout ou partie, sous quelque forme et de quelque manière que ce soit, est interdite sauf accord préalable et écrit de l'éditeur, en dehors des cas prévus par la législation en vigueur en France. Il est précisé que son stockage dans une base de données est également interdit. 


\title{
L'urbanisation rurale en Chine : transformations sociales et effets d'aubaine économiques. Le cas de la cité universitaire Daxuecheng dans la périphérie de la métropole cantonaise
}

\author{
Christophe Guibert*, Benjamin Taunay**
}

La description et l'étude des effets de l'urbanisme sur les populations locales en Chine ne sont pas nouvelles. Souvent, les collusions entre les promoteurs immobiliers et les représentants politiques sont pointées, que ce soit dans les grandes métropoles comme Pékin [Merle, Youjun, 2002 ; Aveline-Duchat, 2013] ou dans les communes rurales. Concernant la cité universitaire Daxuecheng sur l'île de Xiao Guwei, qui est le terrain convoqué dans ce travail, dans la proche banlieue de Canton, d'importantes transformations s'opèrent en un temps court à la suite de l'impulsion volontariste des politiques d'État. C'est justement cet ordonnancement qui permet de justifier l'idée selon laquelle en un même espace physique (le complexe universitaire a été construit sur une île composée de petits villages de paysans) cohabitent ou mieux, se superposent, des populations, des groupes sociaux, aux usages du lieu et aux caractéristiques sociales très différenciés. L'analyse se positionne donc dans l'étude des transformations géographiques, des aménagements produits (sujet sur lequel la littérature scientifique est d'ailleurs conséquente) et dans l'analyse des effets de l'urbanisation rurale ${ }^{1}$ et des « arrangements locaux » [Dorier-Apprill, Jaglin, 2002] inhérents.

Ainsi, en quoi l'urbanisation rurale en Chine transforme-t-elle à la fois l'espace physique (rues, architecture, morphologie urbaine, etc.) et structure-t-elle l'espace social (recrutement social différencié, renversement des structures économiques séculaires) ? Cette question fondamentale, en période d'importantes mutations de

\footnotetext{
* Maître de conférences HDR en sociologie, université d'Angers, UFR ESTHUA tourisme et culture, Laboratoire espaces et sociétés (UMR CNRS 6590).

** Maître de conférences en géographie, actuellement détaché au ministère de l'Europe et des Affaires étrangères, Laboratoire espaces et sociétés (UMR CNRS 6590).

1. Dénomination des échelons urbains (transcription phonétique des mots chinois en pinyin en italique) : province : sheng, municipalité : shi, arrondissement : shiqu, bureau de quartier : jiedao, comité de résidents : junmin weiyuanhui. Dénomination des échelons ruraux : district : qu, bourg : zhen, village : cun, comité des villageois : cunmin weiyuanhui.
} 
la société chinoise, est ici proposée à l'aune d'un cas singulier à proximité immédiate de la ville de Canton. S'il n'est pas question dans cet article d'identifier les stratégies et les enjeux politiques liminaires ayant permis la création et la réalisation d'un complexe universitaire de près de 200000 étudiants en moins d'une décennie, l'analyse des conséquences de l'urbanisation rapide et de la «modernisation des villes chinoises »[Gransow, 2014] met en lumière des effets d'aubaine, des bifurcations biographiques, des transformations sociales, etc. pour le moins structurants. Il en résulte, tant sur le plan spatial que social, un « contexte d'effacement progressif des distinctions entre zones rurales et zones urbaines » [Zhu, Lin, Lin, Chen, 2013].

Le questionnement central de ce texte peut se formuler ainsi : en quoi la transformation fondamentale des propriétés sociales des populations résidentes, à la suite de la venue de nombreux migrants chinois d'une part, attirés par la manne économique escomptée, puis des étudiants d'autre part, permet-elle d'avancer l'idée de contrastes sociaux ? En quoi ces derniers sont-ils potentiellement aux fondements d'un nouvel ordre social au sein des villages ainsi métamorphosés ? Trois parties composent cet article. La première vise à préciser - à partir de l'exemple de la nouvelle cité universitaire Daxuecheng - le cadre politico-juridique et les agencements administratifs ayant favorisé les processus très rapides d'urbanisation rurale en Chine. L'existence de plusieurs villages séculaires sur l'île pose le problème des aménagements et des transformations spatiaux. À ces derniers correspond une double refonte de l'économie locale avec de nouvelles stratégies économiques de la part des villageois d'une part, puis l'arrivée de migrants chinois venus par effet d'aubaine d'autre part. Cette mutation économique du village, analysée dans la seconde partie, a également pour effet différentes formes de ségrégation. Enfin, la dernière partie aura pour objectif de démontrer que la venue de milliers d'étudiants sur l'île, d'une part, et le maintien de quelques villages, d'autre part, a pour conséquence un bouleversement des usages sociaux des lieux.

\section{Cadrages méthodologiques : enquêter en Chine}

Les matériaux empiriques, principalement de type qualitatif, reposent sur plusieurs phases de recueil de données. Une première étape «exploratoire » a été menée pendant une semaine en octobre 2011, elle a été complétée par deux temps d'approfondissement des hypothèses et de recueil de données qualitatives, soit cinq jours en 2013, puis une semaine supplémentaire en mai $2014^{2}$. Chaque période a permis, à la suite des recadrages et des reformulations, d'affiner et de tester des hypothèses. Les données reposent sur des matériaux qualitatifs - entretiens exploratoires et semis directifs menés en mandarin, puis observation - d'une

2. La dernière phase de terrain a bénéficié d'un financement «Mobilités Arianes » du conseil scientifique de l'université d'Angers. 
part et sur un recueil systématique de documentations (de première et seconde main) relatives à l'objet d'autre part.

L'observation non participante, afin de saisir l'ambiance des lieux, l'absence ou la présence d'interaction sociale, les organisations spatiales, etc. a été également largement éprouvée. Par observation, nous entendons la mobilisation de tous les sens du chercheur, «à commencer par la vue et l'ouïe pour enregistrer les pratiques gestuelles et les discours » [Arborio, Fournier, 1999], mais aussi pour être attentif à la présence et à l'agencement des objets, à ce « social objectivé », « social qui s'est cristallisé dans des objets » [Pinçon, Pinçon-Charlot, 1997]. Sans céder à un empirisme naïf, « l'enjeu est bien de rencontrer la réalité concrète de la vie quotidienne » [Piette, 1996]. Une analyse systématique (quantifiée et spatialisée) des traces objectives, mais aussi symboliques de la transformation des villages pris pour objet a été assurée à l'aide de carnets de terrain. Aussi, au-delà d'échanges parfois peu formalisés, néanmoins structurés par les hypothèses de recherche, et une vingtaine d'entretiens (avec des vendeurs dans les échoppes, des gérants ou gardiens de pension, des habitants, des étudiants, des élus des villages puis des fonctionnaires en charge de l'urbanisme), l'utilisation de la photographie, eu égard à «sa force représentationnelle et analytique » [Piette, 2007] est, dans ce texte, convoquée aux fins de description et de preuve à l'argumentation « en apportant des éléments du monde visible qui aident à mieux comprendre une description » [Conord, 2007].

Nous avons sélectionné deux villages sur les cinq que compte l'île, en raison de leur localisation, de leur taille et, surtout, de leur proximité immédiate avec les récents établissements et bâtiments universitaires. Nous avons approfondi en 2013 et 2014 le cas de Beiting cun (initialement exploré en 2011) et engagé l'analyse de Nanting cun cette dernière année à des fins de comparaison. Concernant le premier village, un décompte exhaustif de l'ensemble des pensions et des commerces (coiffeurs, supermarchés, imprimeries, vente de fruits, restaurants chinois et étrangers, etc.) a été réalisé afin de mesurer spatialement la profondeur des transformations apportées au lieu par l'arrivée de résidents extérieurs.

\section{L'urbanisation rurale de Daxuecheng: un cas parmi d'autres ?}

L'urbanisation rapide en Chine et les transformations sociales induites pour les populations locales rurales constituent des objets largement traités en sociologie, en géographie et en science politique ${ }^{3}$. Quelles ont été les grandes étapes de ces mutations et comment ces processus récents, produisant des «formes urbaines spécifiques » [Roulleau-Berger, 2011], conduisent-ils au «miracle de planification » tel que celui de l'île de Xiao Guwei?

3. Il n'est pas ici question de mentionner une succession de recherches : l'article de Chen Yingfang [2009] liste de nombreux travaux pertinents en la matière. 


\section{La cité universitaire Daxuecheng ou l'urbanisation rurale de la périphérie de Canton : " un miracle de planification »}

La ville de Canton, chef-lieu de la province du Guangdong, et ses périphéries constituent un cas remarquable illustrant l'urbanisation chinoise. Principale porte d'entrée touristique en Chine dans les années 1980, la croissance de l'espace urbanisé de Canton a été concomitante d'un renforcement qualitatif des réseaux urbains en vue d'accueillir les Jeux olympiques d'Asie en 2010. Cette stratégie politique s'inscrit dans le droit fil des préconisations du dixième plan quinquennal de la ville 2001-2005, insistant sur l'importance de : «prendre une approche progressive » (хипхи jianjin), d' « économiser les terres» (jieyue tudi), d'adopter un « développement intensif » (jiyue fazhan) et une «planification scientifique » (heli buju). Selon le gouvernement de la ville de Canton, l'action s'est dès lors fondée sur quatre objectifs : «améliorer l'environnement habitable»; «économiser les terres »; «valoriser et diversifier la fonction urbaine »; «établir une nouvelle carte de visite et faire rayonner l'image culturelle et historique de la ville». Sous le slogan performatif de «modernisation» affirmé par les autorités politiques locales, afin de permettre de construire des immeubles d'habitations moins insalubres et surtout économiquement plus rentables, plusieurs quartiers sont ainsi détruits dans et autour de la ville, conduisant à l'expropriation des habitants ainsi que leur relogement dans des zones périphériques. Sous l'autorité du gouvernement municipal, la politique d'urbanisation, en vue des Jeux olympiques d'Asie, intitulée «Habiller et coiffer» (chuanyi daimao), suscita ainsi chez les habitants expropriés une vague de contestation engendrée par la rupture de sociabilités anciennes et l'abandon forcé des usages traditionnels des quartiers.

Ce phénomène est assez bien documenté dans les quartiers centraux de la ville $^{4}$, il l'est toutefois moins dans ses périphéries, elles aussi soumises à une importante urbanisation rurale. La volonté de l'État chinois consiste à produire de l'urbanité dans les zones rurales avec un «effacement progressif des différences entre zones urbaines et zones rurales, particulièrement dans les régions côtières très développées » $[\mathrm{Ibid} .]^{5}$. Dans le cas des périphéries des plus grandes villes du pays, l'urbanisation rurale a produit des «villages urbains » [Li, 2008] ou des « villages dans la ville » [Chung, Hunger, 2013] localisés au sein d'espaces urbains récemment créés. Cette «urbanisation in situ» [Zhu et al., 2013] est clairement observable dans les périphéries des plus grandes villes des provinces du Fujian et Guangdong. Elle demeure toutefois relativement invisible dans les statistiques officielles qui séparent arbitrairement les zones urbaines et rurales, passant sous silence les transitions urbaines en cours dans les espaces ruraux $[\mathrm{Ibid} .]^{6}$. Les processus à l'origine de ces situations spatiales (l'implantation à la campagne

4. Ce phénomène est plus généralement documenté en géographie, en aménagement et en urbanisme de langue anglaise, grâce au groupe de recherche « The China Urban Research Network », regroupant des chercheurs du monde entier.

5. Voir également le numéro de la revue Perspectives chinoises [2013] consacré à ce thème.

6. Zhu Y. [2013] travaille à l'échelle de la province du Fujian à l'aide du recensement de la population (2000), d'annuaires statistiques du Fujian et de l'annuaire socio-économique de la province. Cette approche 
d'entreprises d'État ou des investissements directs étrangers), dans certaines provinces dès le début des années 1980, sont largement documentés, la force du changement de l'espace physique et social par l'implantation d'un complexe universitaire à proximité d'une très grande ville (Canton) l'est-elle beaucoup moins.

Situé dans l'arrondissement de Panyu, sur l'île de Xiao Guwei, au sud de la ville historique de Canton, le complexe universitaire Daxuecheng regroupe près de 200000 étudiants au début des années 2010, alors que le début de sa construction dans sa première phase date seulement de 2004 (les premiers cours ont été assurés en septembre 2005). Le projet a débuté en octobre 2000, sous l'impulsion de la municipalité de Canton (avec un premier document intitulé «Comparaison des zones pour établir le nouveau complexe universitaire », guangzhou xin daxuecheng yuanqu xuan zhifang an bijiao yanjiu), puis la publication officielle du projet dans un discours conjoint du secrétaire du Parti communiste de la municipalité et du maire de la ville dès le 6 février 2001. Une carte a été diffusée par le comité du Parti communiste de la ville de Canton le 2 mars 2001 à l'ensemble de la population locale. D'un statut rural, les terres de l'île, historiquement sous autorité du district, sont dorénavant placées sous la compétence de l'arrondissement de Panyu, qui fait partie intégrante de la ville de Canton. Une planification complète de l'île en cinq grands ensembles universitaires (zu tuan) est réalisée, dont quatre sont directement positionnés à l'endroit des anciens villages. Les anciennes terres agricoles sont destinées à l'établissement des différentes universités tandis que les agglomérations villageoises demeurent sous la responsabilité des comités de villageois : les cinq villages subsistent en effet dans le schéma directeur initial [Zhong, 2007].

Les grandes infrastructures universitaires construites par l'État se sont installées sur les terres agricoles expropriées (les habitants des villages voisins ont perdu le droit de cultiver les terres sur ordre de la municipalité, au nom de "l'intérêt public»). Ne demeurent des anciennes formes spatiales (villages et champs) que ces cinq villages - seuls témoignages des négociations ayant eu lieu entre les communautés villageoises et les collectivités locales lors de l'élaboration du schéma directeur - qui s'insèrent de facto dans le tissu urbain (encadré 1). En lieu et place d'anciens champs, 310 bâtiments ont été construits, pour une superficie bâtie totale de $5200 \mathrm{~km}^{2}$, ainsi que 70 kilomètres de routes de niveau municipal damées et 120 kilomètres de routes et chemins au sein des complexes universitaires. Des quartiers résidentiels (xiaoqu) ont également été construits au cœur de l'île. Le projet a coûté (fin 2005, année de début des enseignements)

\footnotetext{
à grande échelle permet de mesurer la part de la population «quasi urbaine » oubliée (« invisible » selon l'auteur) dans les statistiques officielles. Une telle approche quantitative n'a pas été possible dans notre cas d'étude, l'accès aux sources officielles ne nous ayant pas été autorisé lors de nos passages (2013 et 2014) dans les différents départements traitant de l'aménagement à l'échelle du bureau de quartier (jie dao). Ce constat témoigne du fait qu' "entrer dans des places» et s'inscrire dans une logique de "régime de confiance » n'est pas toujours aisé en Chine [Roulleau-Berger, 2012]. Notre travail part de fait d'une situation observable (l'urbanisation in situ d'une île définie comme espace rural) pour en expliquer les logiques contemporaines à l'aune de processus économiques (la transformation des habitats et leur système d'exploitation) et sociaux (les contrastes d'utilisation de l'espace public).
} 
20 milliards de yuans (environ 2,4 milliards d'euros) pour la construction de dix composantes universitaires, mobilisant 5327 ouvriers et ingénieurs chinois et étrangers.

\section{Encadré 1 - Les aménagements et les constructions du complexe universitaire :} accueillir 200000 étudiants

Que ce soit les immeubles destinés à l'hébergement des étudiants, les établissements des universités ou les complexes sportifs à disposition des étudiants, la transformation de l'île a été spectaculaire en une décennie. En 2014, seules les zones historiquement occupées par les cinq villages (Beiting, Beigang, Nanting, Suishi, Lianxi) témoignent de l'organisation spatiale précédant la construction de Daxuecheng. L'île est maintenant complètement intégrée au tissu urbain de la ville de Canton, accessible par deux lignes de métro ainsi que par une autoroute intra-urbaine. Le front pionnier de l'urbanisation se situe au niveau de la gare de trains à grande vitesse, la gare du Sud, à $10 \mathrm{~km}$ environ au sud de l'île.

Une partie du complexe sportif, situé au centre de l'île, est positionnée à égale distance de la plupart des universités (photographie 1). Le centre d'exposition scientifique (photographie 2) et une partie des bâtiments proches de Beiting (photographie 3) témoignent de l'envergure architecturale des complexes universitaires.

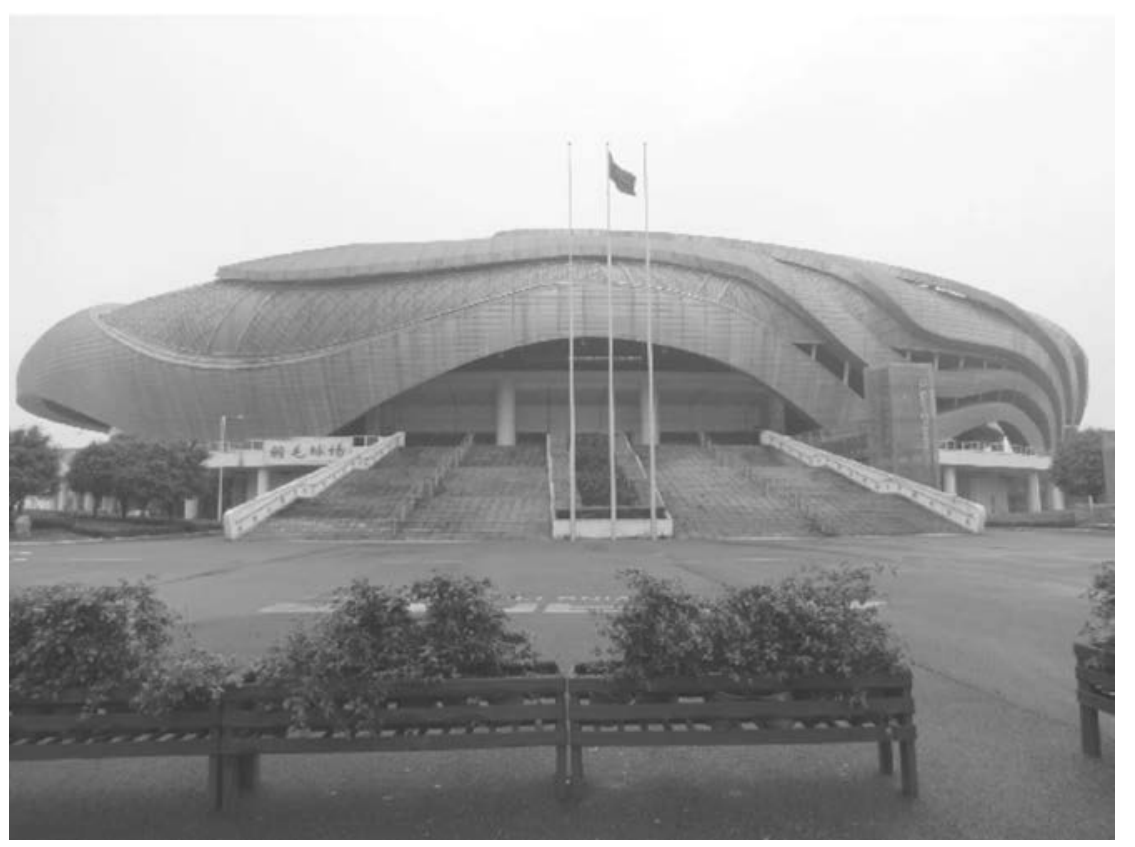



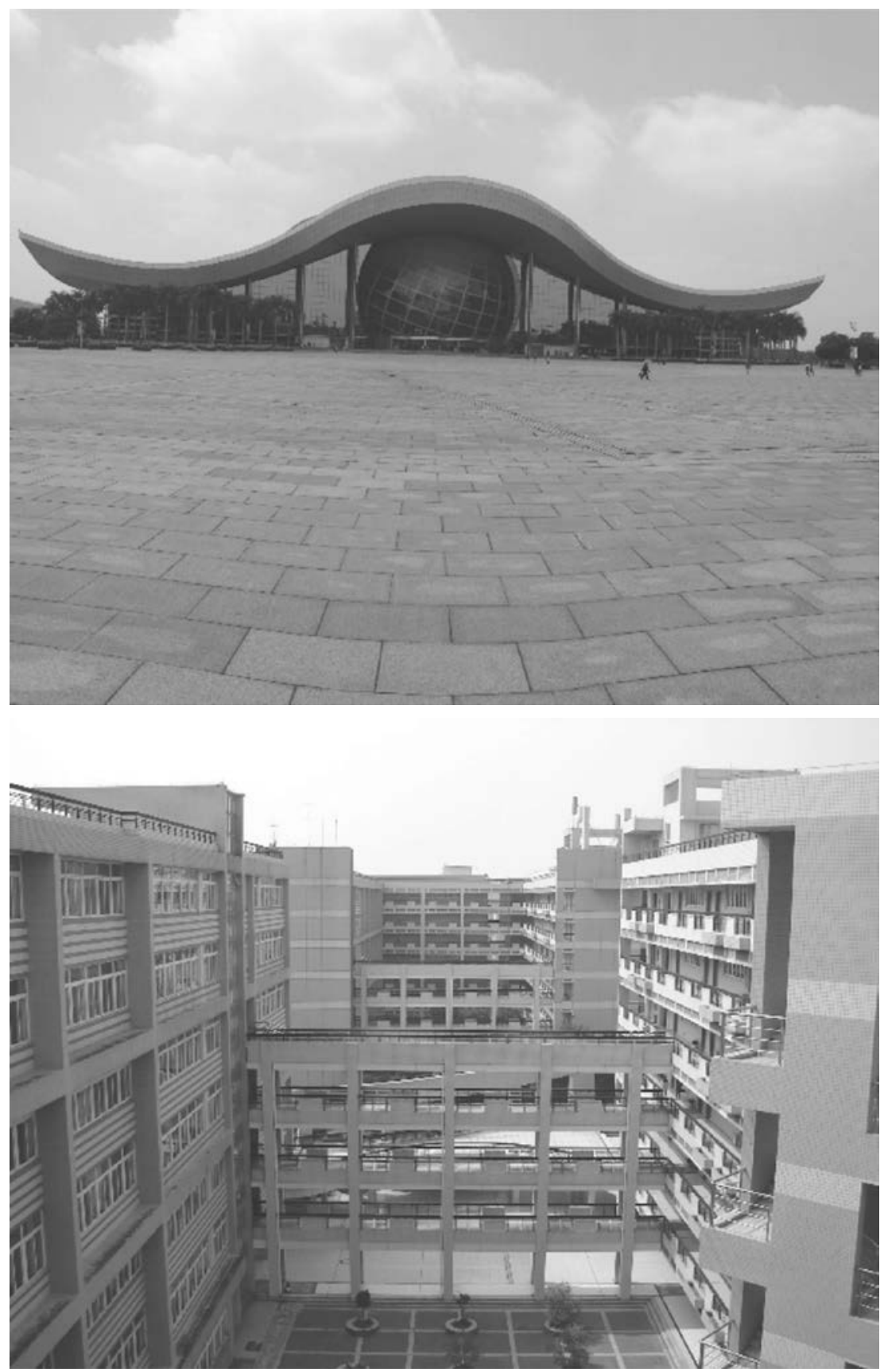

Contiguës au village de Beiting - à l'ouest -, les dernières parcelles dédiées aux cultures par les villageois (photographie 4 ; 2013) contrastent avec les bâtiments nouvellement construits. Quant aux axes routiers créés au sein de l'île pour relier les complexes universitaires entre eux grâce à un important réseau de bus ainsi qu'à Canton (photographie 5), ils contribuent à isoler et enclaver les villages. 


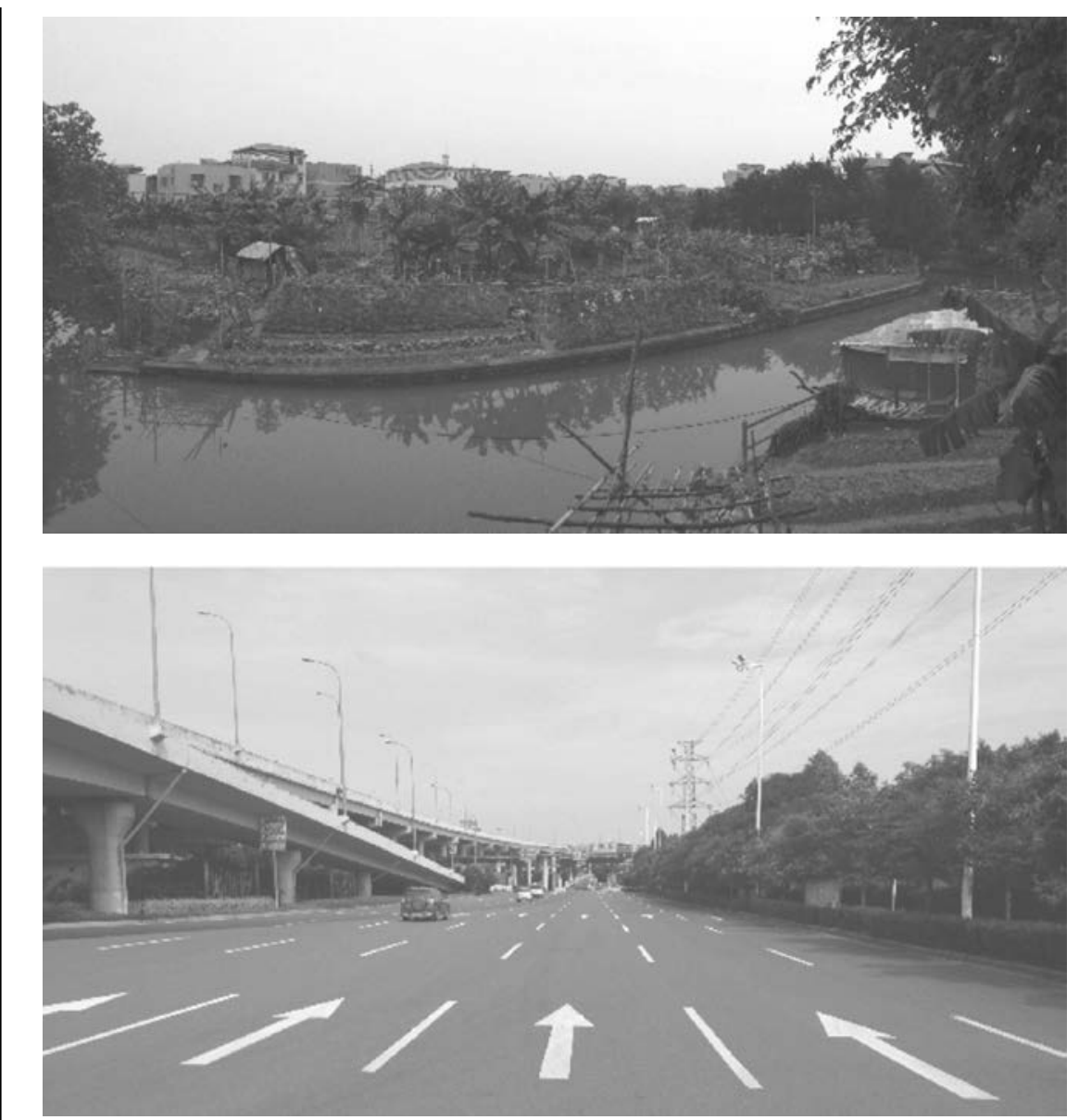

Photographies : auteurs, 2011, 2013, 2014.

\section{Les villages au sein de Daxuecheng : des isolats face à l'urbanisation}

À la suite de la construction des complexes universitaires (facultés, cités universitaires), les villages ont rapidement été circonscrits dans des limites spatiales bornant les habitations et les rues séculaires par les grands axes routiers. L'organisation des seizièmes Jeux asiatiques à Canton, du 12 au 27 novembre 2010, a cependant constitué une étape importante dans l'urbanisation de l'île. Manifestation d'envergure faisant participer des sportifs asiatiques et du Moyen-Orient tous les quatre ans depuis le début des années 1950, les Jeux asiatiques sont en quelque sorte des Jeux olympiques à l'échelle du continent. Plus de 2500 athlètes de 41 nationalités différentes ont participé aux Jeux de Canton, largement dominés, sur le plan sportif, par la délégation chinoise. À l'instar de Pékin (Jeux olympiques en 2008) ou Shanghai (Exposition universelle en 2010), Canton apparaitt ainsi tels une «ville-monde » [Gransow, 2014], un centre métropolitain, économique et 
culturel où les effets de la production de l'espace urbain sont prégnants auprès de la population locale. Les autorités locales ont dès lors décidé de dissimuler les villages à proximité des principaux axes routiers à l'aide de grandes palissades en tôle. Même si les barrières ont été détruites en 2015 après cinq années d'existence, la mise à l'écart des villages, tant physiquement que symboliquement, caractérise une urbanité singulière: entre les routes à six voies (photographie 5) et les complexes universitaires imposants, les villages sont des isolats positionnés au milieu d'infrastructures récentes.

Le mouvement d'urbanisation de la Chine, de manière générale ou plus précisément dans le cas de l'île de Xiao Guwei, peut se résumer par la formule « aucune force ne peut s'opposer aux bulldozers » [Yingfang, 2009], que ces derniers soient bien réels ou, métaphoriquement, politiques. Dans notre cas d'étude, les sites des villages ont été conservés au sein du plan d'aménagement directeur de l'île de Xiao Guwei, mais les villages ont toutefois été physiquement et symboliquement condamnés entre les axes routiers reliant les universités entre elles. Le village de Beiting illustre bien ce processus de mélange de conservation à l'intérieur des frontières du village et, concomitamment, d'exclusion puis de mise à l'écart. Le droit d'utilisation du foncier (construction, revente des bâtiments) revient toutefois de droit aux comités de villageois, ces derniers décidant ainsi de rester ou non sur place, ce à quoi le gouvernement du bureau de quartier de Xiao Guwei ne peut s'opposer. La possibilité de rester est, pour l'instant, acquise aux paysans. Toutefois, «la vie n'est pas facile » selon le maire du village de Beiting qui, fataliste, déclare clairement regretter la situation antérieure. Les revenus des villageois ont été nettement amputés et nombre d'entre eux ont quitté le village dès le début des travaux d'aménagements de l'île :

Avant la construction de Daxuecheng, notre village comptait 4300 habitants, mais maintenant 2100 ont déménagé hors d'ici, presque la moitié. C'était en 2003 notamment quand les travaux ont débuté et que les destructions ont commencé. Les habitants qui ont déménagé sont partis au bourg de Xinzao, au nouveau village de Guwei. [...] Avant la construction de Daxuecheng, la vie était plus simple. À ce moment-là, on pouvait cultiver la terre et vivre ici indépendamment. Maintenant, il faut aller chercher un travail. La vie n'est pas facile. Autrefois, nous avions des terres et des champs, chacun pouvait labourer et cultiver, chacun pouvait vendre, nous étions indépendants. Maintenant que nous n'avons plus de terres, nous ne pouvons plus labourer, il faut aller chercher du travail. Autrefois, une personne gagnait environ 8000 yuans par an, maintenant on a 3000 yuans, ce n'est pas suffisant ${ }^{7}$.

L'urbanisation accélérée n'a-t-elle toutefois que des effets « destructeurs »? Si le départ d'une partie des habitants fait écho à la construction d'établissements universitaires, privant les paysans de leurs espaces de productions agricoles, l'arrivée d'étudiants engendre, en miroir, l'émergence rapide d'une diversification économique et professionnelle.

7. Entretien effectué à la mairie de Beiting, mai 2014, dans un bureau collectif. Le maire nous a reçus à la suite d'une réunion du comité de villageois où il était question de compensations financières du gouvernement : «le parti doit garantir notre niveau de vie », précise-t-il à ce propos. 


\section{Les transformations de l'économie et les nouvelles stratégies économiques}

Beiting compte 2000 habitants originaires du village pour 4213 migrants venus d'autres régions de Chine (source : Comité de l'île de Xiao Guwei, 2012), soit 2,1 habitants extérieurs pour 1 villageois autochtone. Comment se traduit spatialement ce déséquilibre ? Quelles incidences cela a-t-il en termes économiques et sociaux?

\section{Construire des pensions : la stratégie pragmatique des villageois}

Hormis les infrastructures universitaires, l'ensemble des nouveaux services à destination des étudiants a été créé au sein même des villages, en lieu et place d'habitations réhabilitées pour l'occasion. En raison de la nature rurale des villages qui, contrairement aux terres agricoles expropriées (devenues juridiquement résidentielles et urbaines) dont ils ont perdu l'exploitation, une gouvernance particulière s'y est installée. Les habitants qui y disposent toujours de hukou ruraux peuvent gérer eux-mêmes l'espace dont ils ont la jouissance, soit en construisant eux-mêmes de nouveaux bâtiments, soit en octroyant ${ }^{8}$ la gestion de ceux-ci à des populations migrantes (possédant elles aussi un permis de résidence rural). Le village de Beiting compte ainsi en 2014, 158 pensions au total, positionnées selon une logique spatiale - deux polarités - singulière. Plus de la moitié (53,8 \%, soit 85 établissements) est localisée face à l'Université de Canton au sud du village, alors que $38 \%$ (60 pensions) sont orientées vers l'Université Normale du Sud de la Chine à l'est du village. Cette forte concentration a des incidences sur les prix de l'immobilier, comme en témoigne un gérant d'une pension en 2014 : «Plus on est loin d'une entrée du village, moins c'est cher. Les prix vont de 1 à 3 ! » ${ }^{9}$. Preuve de l'importance de la présence d'étudiants et du ciblage de « clientèle », de nombreuses locations de vélos dans la partie du village faisant face à l'Université Normale du Sud sont proposées. Un grand nombre de restaurants, de plein air ou couverts, avec plusieurs centaines de places, atteste manifestement de stratégies entrepreneuriales spécifiques : ce sont les étudiants ${ }^{10}$ qui font principalement vivre économiquement les villages.

Les pensions sont construites par les villageois à partir d'habitations existantes (encadré 2). Ces derniers, abandonnant leur métier d'agriculteur, deviennent gérants ou, conservant leur profession d'origine, louent la gérance à des migrants chinois. La stratégie est double : accumuler du capital économique en louant des chambres à des étudiants, mais aussi agrandir les surfaces habitables et ainsi espérer des

8. De manière illégale, ce qui est une des sources de tension entre les villageois et les autorités du Canton chargées de la planification et l'aménagement de l'île.

9. Entretien effectué le 29 mai 2014. Plus loin dans l'entretien, l'interrogé demande combien nous avons payé pour le coiffeur; à notre réponse (20 yuans), il répond «nous, on connaît les endroits à 10 yuans ».

10. Les étudiants ne sont pas les seuls nouveaux arrivants dans le village, des salariés aux revenus modestes (moins de 2000 yuans) viennent également profiter d'opportunités de logement à prix réduit, comme l'indique le Quotidien de Canton [2010]. 
compensations financières plus élevées de la part du gouvernement en cas de destruction du village : "La finalité des expulsions est acceptée sous condition d'en marchander les modalités d'application » [Laurans, 2006]. Le montant des compensations s'applique en fonction de la période d'entrée dans l'habitation. Plus un villageois habite depuis longtemps une maison, plus la compensation financière est potentiellement importante. La moitié des habitants autochtones est ainsi partie du village dès les premières années de la construction du complexe universitaire. M. Wang, en charge des négociations avec les habitants des villages (en poste au bureau de quartier de l'île) déclare toutefois que, «au début de la construction de Daxuecheng, les choses étaient pressées, les relations entre le gouvernement et les villageois n'étaient pas très bonnes. Aujourd'hui encore, les villageois proposent des conditions, nous voyons si nous pouvons y répondre favorablement. Nous négocions maison par maison ${ }^{11}{ }^{~}$. Plus tard dans l'entretien, il précise, assez énervé, que « certaines de leurs (les habitants du village) exigences sont trop élevées, si nous les acceptons, cela peut causer une injustice dans la destruction et le déplacement des habitats ! » Les habitants qui n'ont pas accepté les conditions initiales du gouvernement local ont depuis continué à négocier, voyant la valeur de leur bien augmenter avec l'arrivée des étudiants dans le village «Une fois que Daxuecheng a été achevé, les villageois ont petit à petit construit beaucoup de maisons, pour les louer. Ainsi ils gagnent plus que s'ils vendaient leurs terrains pour aller habiter dans le nouveau quartier. » Le maire de Beiting indique que les revenus s'élèvent, " pour ceux qui ont des chambres à louer, à environ 3000 yuans par mois par foyer ». Ces effets d'aubaine professionnelle et ces arrangements opportunistes, grâce à la présence de nombreux étudiants, semblent toutefois s'inscrire dans l'illégalité selon le maire. Les effets pourraient être la destruction pure et simple des habitations nouvellement édifiées :

Ces gens construisent illégalement, ils pillent! Le gouvernement n'arrive pas à contrôler. À l'origine, la terre de ces villageois est la propriété du comité des villageois, le gouvernement n'a pas les moyens de contrôler. Tous ces bâtiments sont illégaux, les habitants les louent, mais ce n'est pas légal. Donc maintenant, le gouvernement a déclaré trois mesures politiques pour mettre progressivement en échec ces constructions et les détruire. Le gouvernement a déjà commencé à faire le tour des bâtiments, mais les mesures n'ont pas encore été promulguées.

\section{Encadré 2 - Un village en reconstruction}

Dans la plupart des rues, trop étroites pour accueillir des voitures, des échafaudages sont disséminés (photographie 6). De nombreuses maisons d'habitations sont soit rasées et reconstruites, soit aménagées de telle sorte qu'un ou deux étages supplémentaires puissent être réalisés. Ces habitations traditionnelles sont celles de Chinois habitant le village avant que les campus ne viennent déclencher des processus de plus-value immobilière et de pression foncière. Les traces d'habitat ancien deviennent dès lors exceptionnelles même si une première et timide politique de conservation a été promulguée en 2012 par la mairie.

11. Entretien effectué le 12 avril 2013. Le nom a été modifié. 


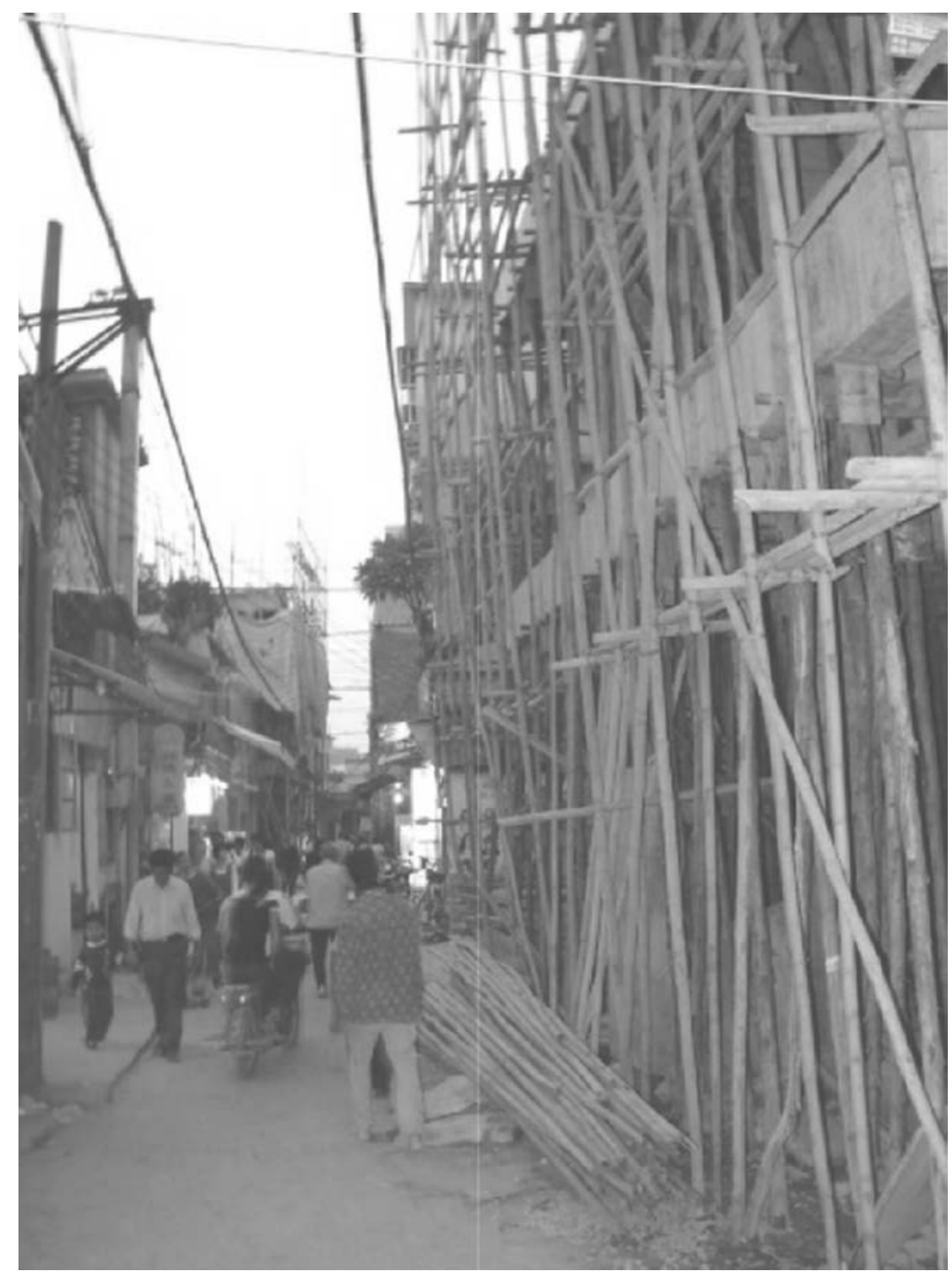

Photographie : auteurs, octobre 2011.

\section{Effets d'aubaine et persistance des ségrégations}

L'arrivée massive d'étudiants a créé une nouvelle organisation économique dans ces «villages urbains » [Roulleau-Berger, 2011; 2013 b] enclavés dans le complexe universitaire de l'île. Les migrations ont pour effet des inégalités économiques et professionnelles. De nouveaux services, à destination d'une clientèle d'étudiants principalement, ont peu à peu été proposés. Ceci a été possible parce 
qu'une partie des terres anciennement agricoles « appartient à la collectivité des villageois, l'autre à l'État » selon M. Wang. Comme ailleurs dans la province du Guangdong, de «petits groupes de villageois » (cunmin xiaozu) ont conservé « la propriété de la terre et les familles membres furent autorisées à cultiver la terre qui leur avait été attribuée sans contrepartie financière ». [Chung, Hunger, 2013] L'expropriation sur ordre d'intérêt public sur ces terres à statut rural n'a donc pas été possible partout, obligeant les autorités à négocier et à développer une gouvernance particulière, adaptée à chaque situation locale.

L'amputation d'une partie des terres villageoises (celles dont la propriété est détenue par le gouvernement de Canton) a toutefois forcé une partie des habitants du village à développer de nouvelles stratégies. La structure sociale de la population des villages a en conséquence, à l'aune de ces « bifurcations biographiques » [Roulleau-Berger, 2013 a], été largement modifiée. La plupart des nouveaux commerces (pensions, salons de coiffure, restaurants, supérettes, magasin de prêtà-porter et reprographie) sont gérés par des Chinois qui ne sont pas des villageois autochtones. Des paysans propriétaires de leur terre louent leur maison à des migrants là où d'autres ont choisi de déménager, en laissant toutefois la gestion de pension à un membre de leur famille. Malgré la possibilité d'obtenir de nouveaux revenus en restant au village, les conditions de vie sur place sont loin d'être idylliques ${ }^{12}$. Ce processus participe à la reconfiguration économique, urbaine et sociale des villages de l'île. Ces Chinois d'autres provinces ont connaissance des opportunités d'emplois par le réseau (amis, famille) : «Ce sont les réseaux de solidarité et d'entraide entre migrants issus de la même région qui vont favoriser la production et la reconnaissance de compétences et d'expériences » [RoulleauBerger, Lu, 2005]. Les sites internet dédiés aux offres d'emploi sont également mobilisés par les migrants les plus jeunes. Ces perspectives économiques sont perçues comme de véritables effets d'aubaines pour des Chinois soit diplômés, mais sans emploi dans leur région d'origine, soit anciens agriculteurs et ouvriers souhaitant arrêter des professions pénibles physiquement et financièrement peu intéressantes. Les migrations spatiales, en lien avec les structures traditionnelles de la société chinoise telles que les systèmes de parentés permettent ainsi les mobilités professionnelles et sociales chez les Chinois provenant d'autres régions. Ces migrations ne garantissent toutefois pas mécaniquement une hausse de revenus par rapport à leurs anciens emplois respectifs.

Bien que de nouveaux arrivants s'installent dans les deux villages investigués pour y développer des affaires et profiter de la manne économique des étudiants, il n'en demeure pas moins que ces commerçants et hôteliers ne jouissent pas des mêmes droits juridiques et avantages économiques que les habitants installés avant le début de la construction de Daxuecheng. Ces habitants temporaires louent aux résidents permanents les bâtiments qu'ils exploitent commercialement (pensions, magasins), conduisant à des conditions de vie pour le moins économiquement

12. Nous n'avons toutefois pas pu quantifier la proportion des personnes ayant choisi l'une ou l'autre de ces stratégies. 
précaires. Gérer une pension implique par exemple de laisser le maximum d'espace pour les chambres destinées aux clients. Pour les gestionnaires de ces établissements, cela conduit parfois à loger à plusieurs dans un réduit. C'est le cas de Wang, neveu du gérant d'une pension dont il assure l'accueil, obligé de dormir avec son oncle sur le lit au milieu d'une chambre qui fait également office de réception de la pension, mais aussi de cuisine, de salle à manger et de séjour, comme en témoigne l'encombrement (ordinateur portable, linge repassé et à sécher, valise, nécessaire de toilette) de la pièce. Trois personnes (l'oncle, son épouse, leur fille) habitent régulièrement cet espace auquel est accolée une petite salle de bains.

Enfin, si les habitants non natifs du village de Beiting sont numériquement majoritaires (plus des deux tiers des résidents non étudiants selon le recensement de la mairie en 2010), une ségrégation sociale s'esquisse à l'aune des droits alloués à chaque catégorie de population. Ils ne disposent pas du droit d'utilisation du sol : leur Hukou - système d'enregistrement de résidences - n'étant pas enregistré dans un des villages de l'île de Xiao Guwei, les nouveaux arrivants ne disposent pas des privilèges sociaux ordinaires dont bénéficient les villageois autochtones (gratuité de l'école primaire, remboursement des frais de santé, aides au chômage). La population venue de l'extérieur de Canton n'a en outre pas d'incidence politique sur les choix et stratégies de développement local comme le confirme Xi J., un «nouveau villageois »: «Je n'ai pas le droit de voter à Beiting, je n'ai pas ce pouvoir, je ne peux voter que dans mon village, là où est mon Hukou [...] Je n'ai pas les mêmes accès aux soins que les autres habitants ${ }^{13}$. » La population migrante a toutefois un impact sur la vie locale en matière d'organisations spatiales, de structures sociales et de développement économique de l'île.

\section{Une structure sociale de la population modifiée}

Quelles formes prennent les processus de cohabitation des populations villageoises et paysannes avec les dizaines de milliers d'étudiants présents sur l'île dont une proportion importante d'entre eux fréquente les villages pour s'y restaurer, acheter, voire s'y loger ? Déambuler dans les villages de l'île situés à proximité des complexes universitaires permet de visualiser immédiatement des usages différenciés de l'espace (notamment dans les rues de Beiting, voir encadré 3). Cette coprésence de fait, improbable et impromptue, est rendue possible ici à cause de la rapidité des transformations spatiales et des modifications de la structure sociale de la population.

13. Entretien effectué le 23 mai 2014. 


\section{Encadré 3 - Des usages différenciés de l'espace public}

Alors que les étudiants, livres à la main, traversent les quartiers en direction de l'université, des ouvriers vont et viennent également vers les chantiers de réfection des habitations (photographie 7). Les tenues suffisent d'ailleurs à identifier les étudiants et étudiantes (parfois en tenue «légère » et hauts talons) puis les ouvriers, en bleus de travail et tongs, voire pieds nus. L'espace public est ici le lieu de cohabitations éphémères (photographie 8).
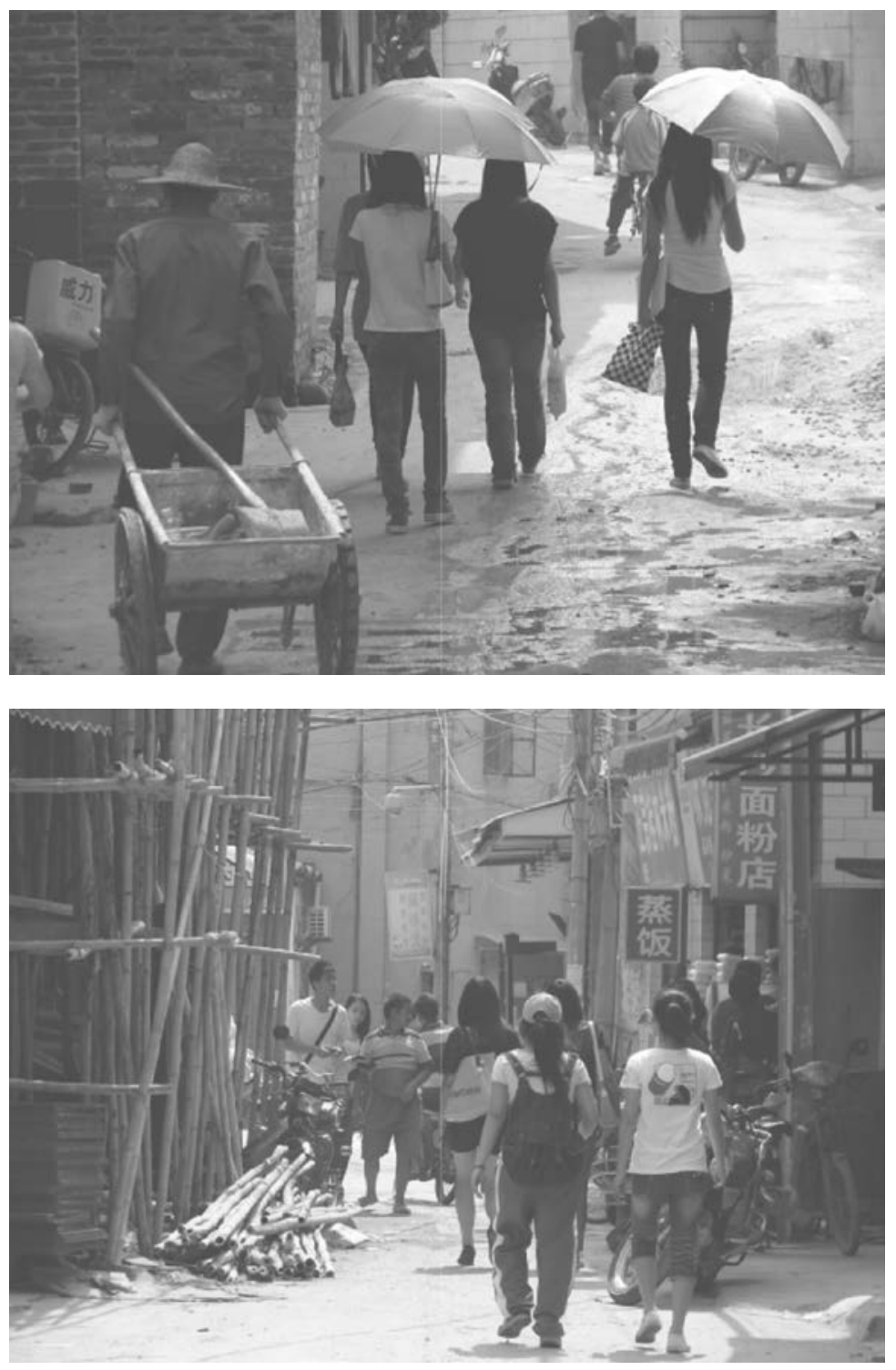


\begin{abstract}
La plupart des croisements de ruelles du village de Beiting sont agrémentés de multiples panneaux indiquant la présence de pensions à destination d'une clientèle estudiantine (photographie 9). Les barrières autour du village deviennent quant à elles le support de publicités diverses à destination des étudiants (emplois temporaires, excursions, rencontres musicales le week-end, etc.; photographie 10).
\end{abstract}

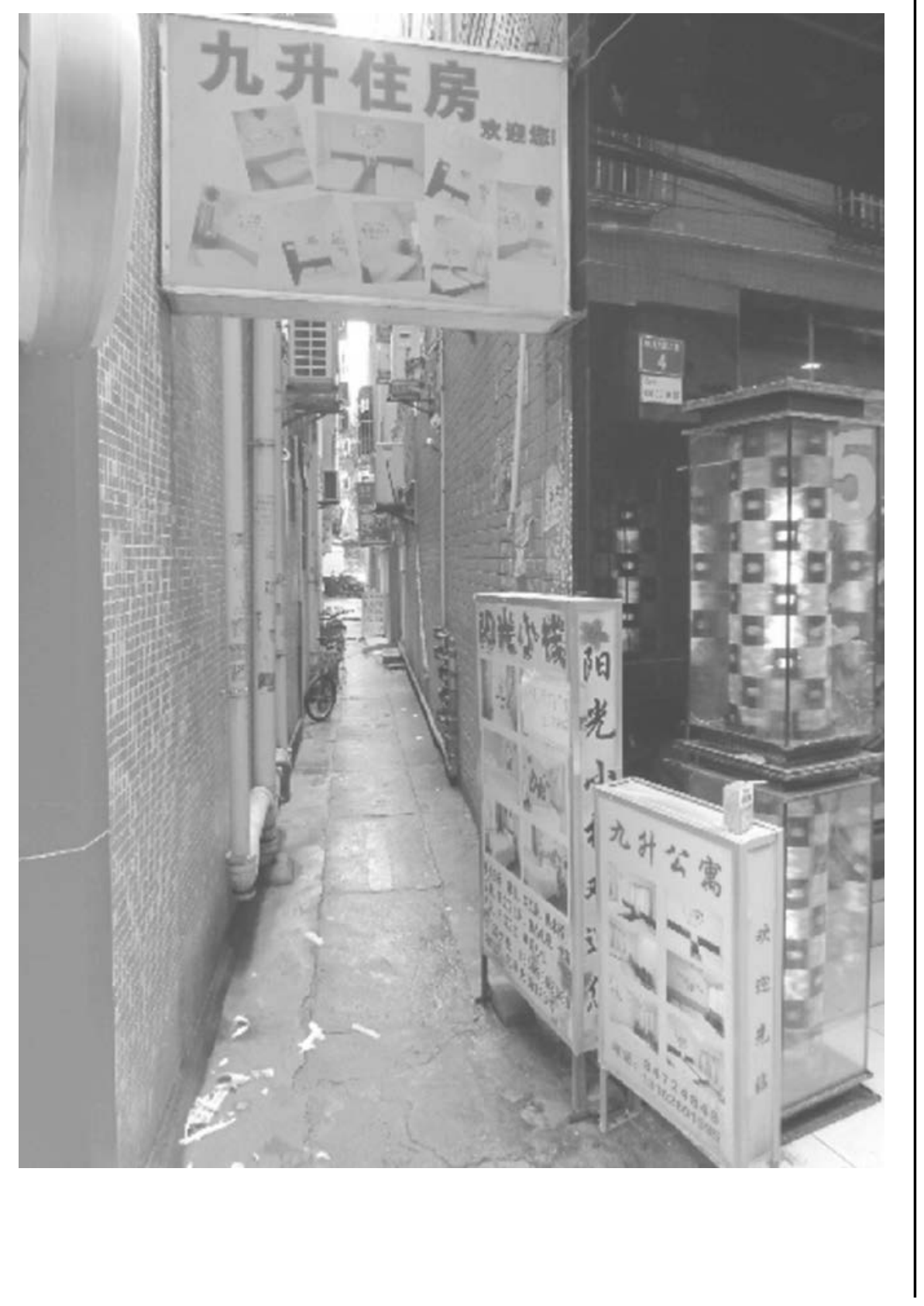




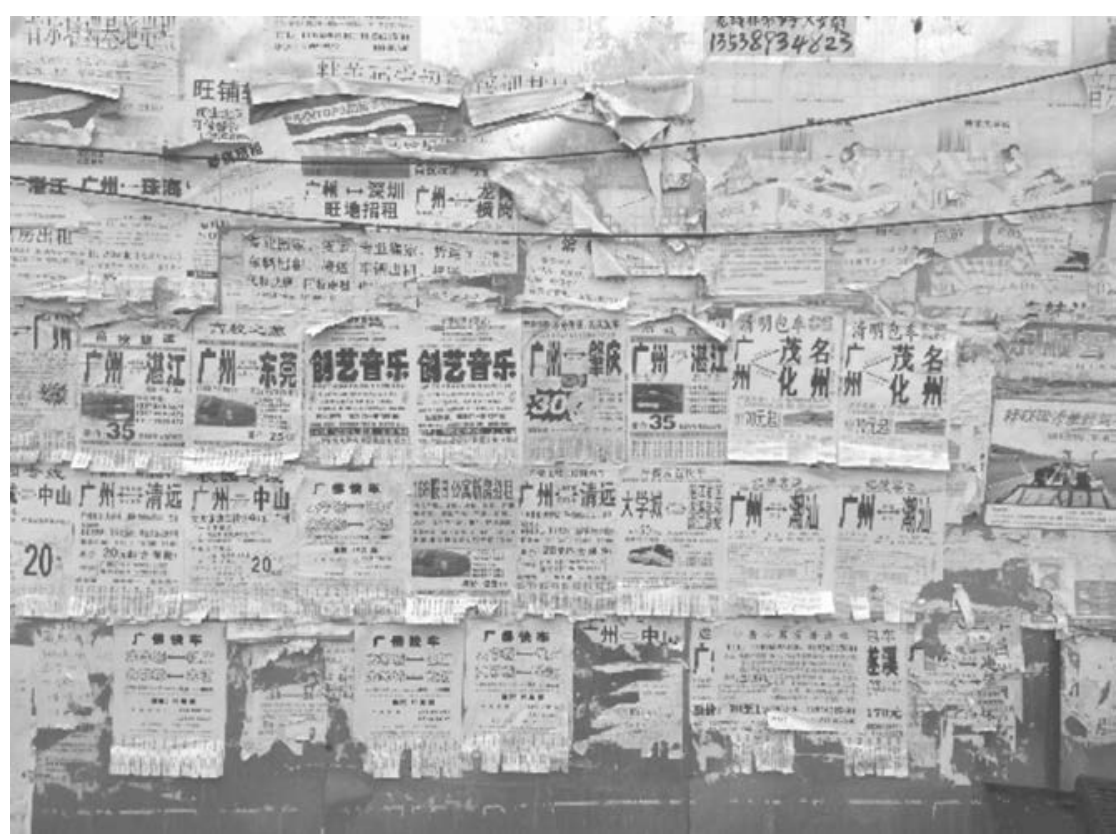

Photographies : auteurs, octobre 2011, avril 2013 et mai 2014.

Les cas présentés ci-après visent à illustrer l'analyse et administrer la preuve que les populations de l'île sont composites et hétéroclites. Les contrastes sociaux observés se déclinent à l'aune des origines sociales et géographiques des individus interrogés.

A - Ranjian W., âgé de 26 ans, a fait des études dans le domaine du design et de la mode vestimentaire dans la province du Jiangxi «à la capitale ». Il a obtenu sa licence à 23 ans. Il est arrivé sur l'île en 2009, en réponse à une offre d'emploi, afin d'occuper le poste de vendeur dans un magasin de vêtements. Il a ensuite ouvert une agence de conseil immobilier par opportunité, « en indépendant » début 2011. Sa clientèle est surtout constituée « de gens qui louent plus que de gens qui achètent des logements ». En 2014, l'agence n'existe plus, témoignant de la fragilité des entreprises quant à leur pérennité d'une part et l'instabilité professionnelle et sociale dans laquelle s'inscrivent certains migrants d'autre part :

« J'ai vu que les prix des maisons augmentaient beaucoup et qu'il n'y avait personne pour vendre les parcelles et les appartements. Les prix ont été multipliés par cinq en six ans ! Je suis le seul à faire cette activité dans le village, car je me suis rendu compte qu'il y avait des besoins des deux côtés : pour les acheteurs, car les procédures d'achat sont compliquées et pour les loueurs qui ont du mal à trouver leurs clients. S'il y a beaucoup de boutiques, c'est qu'il y a de moins en moins d'habitations et de surface utile. Les habitants ont été concentrés ici, à cause de la construction de l'université. Certains ont donc cherché d'autres activités : ils ont 
ouvert des restaurants par exemple. On peut dire que, puisqu'il y a beaucoup d'étudiants, c'est une conséquence : il y a des salons de coiffure, des restaurants, etc. ${ }^{14}$. »

B - Un couple, gérant d'une supérette sur l'île, est quant à lui originaire du sud-est de la province. Avant cette récente activité professionnelle, tous deux étaient ouvriers. Leur enfant est scolarisé dans un lycée de Canton. Le magasin a ouvert au début de l'année 2010 et les heures d'ouverture sont calquées sur les modes de vie de la population étudiante, la supérette est en effet ouverte jusqu'à une heure du matin, chaque jour. Le local qu'ils utilisent était anciennement dédié à un restaurant. Leur affaire, également créée par l'opportunité de l'essor rapide de la population sur l'île, semble être économiquement prolifique : «ça marche bien. On est venu ici parce que la population migrante est très importante. On est venu parce qu'il y a de l'argent à se faire, parce qu'il y a beaucoup de monde. Nos clients, c'est tout le monde, les habitants et les étudiants ${ }^{15}{ }$.

C - Chen M., gérant d'un salon de coiffure (encadré 4), est lui originaire de la province du Hunan. Avant cette activité, il travaillait dans le domaine de l'art et du design. Il a ouvert son entreprise il y a trois ans dans une maison d'habitation : « avant, il n'y avait rien, simplement une maison, des habitants ». Son entreprise, qui emploie 14 salariés, ne semble pas souffrir de la concurrence des nombreux autres salons de coiffure, eu égard au nombre important d'étudiants : « Notre clientèle principale, ce sont les étudiants. Nous ne serions pas venus ici s'il n'y avait pas le complexe universitaire. Nous sommes venus parce qu'il y a des étudiants. Nous n'avons pas de concurrence ici : chaque salon a sa clientèle. Il y a assez d'étudiants dans le village ${ }^{16}$ ! » La multitude de salons de coiffure dans le village témoigne d'une clientèle nombreuse et prête à en acheter les services. Les marques internationales (usurpées, telle «L'Oréal ») sont d'ailleurs bien visibles à l'entrée des salons. Les lumières et jeux de lumière et les sonorisations, largement écoutables de la rue, renforcent la distance symbolique et physique entre la clientèle ordinaire de ces établissements et les ouvriers ou les villageois « traditionnels ». Les coiffures les plus valorisées dont d'ailleurs celles affichées par les coiffeurs et coiffeuses eux-mêmes : décoloration, coupes « à la Coréenne », etc. La «mode étudiante » semble être l'unique stratégie marketing des salons de coiffure.

D - Un autre couple, accompagné de leur fils, encore au collège, gère une entreprise de reprographie et d'imprimerie qui a ouvert en 2006, service ordinaire dans «un quartier étudiant» (encadré 4). Les parents ont suivi leurs études «jusqu'à l'école élémentaire ». Ils sont tous trois originaires de la province du Hunan et, suivant les conseils d'amis, ont profité de cet effet d'aubaine qu'a constitué l'ouverture de l'université. «Nous avons des amis qui nous ont présenté ce projet de ville universitaire, et nous sommes venus. Avant de venir, nous faisions ce métier dans notre région d'origine, mais nous travaillions pour quelqu'un

14. Entretien effectué le 19 octobre 2011 dans l'agence immobilière.

15. Entretien effectué le 19 octobre 2011.

16. Entretien effectué le 19 octobre 2011. 
d'autre. Maintenant, nous travaillons à notre compte et nous sommes très contents d'être ici. Il n'y a pas de concurrence, chacun a sa clientèle. »

\section{Encadré 4 - Des espaces marchands pour étudiants}

Les magasins et services marchands majoritaires dans les rues les plus passagères sont clairement destinés à une clientèle universitaire. Imprimerie et reprographie, librairies universitaires, magasins de prêt-à-porter de type «sportswear » ou à la dernière mode féminine, pâtisseries « haut de gamme », etc.

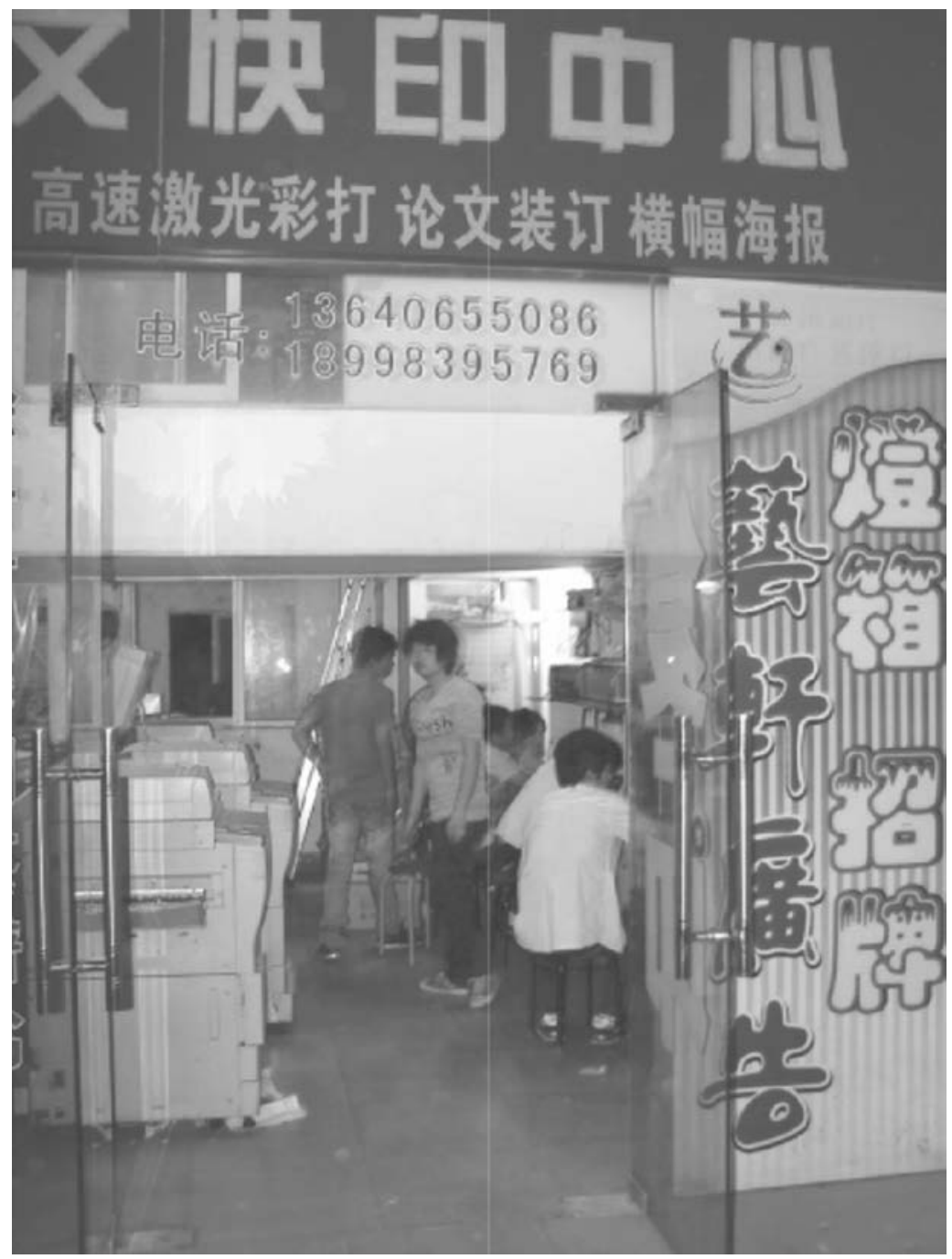




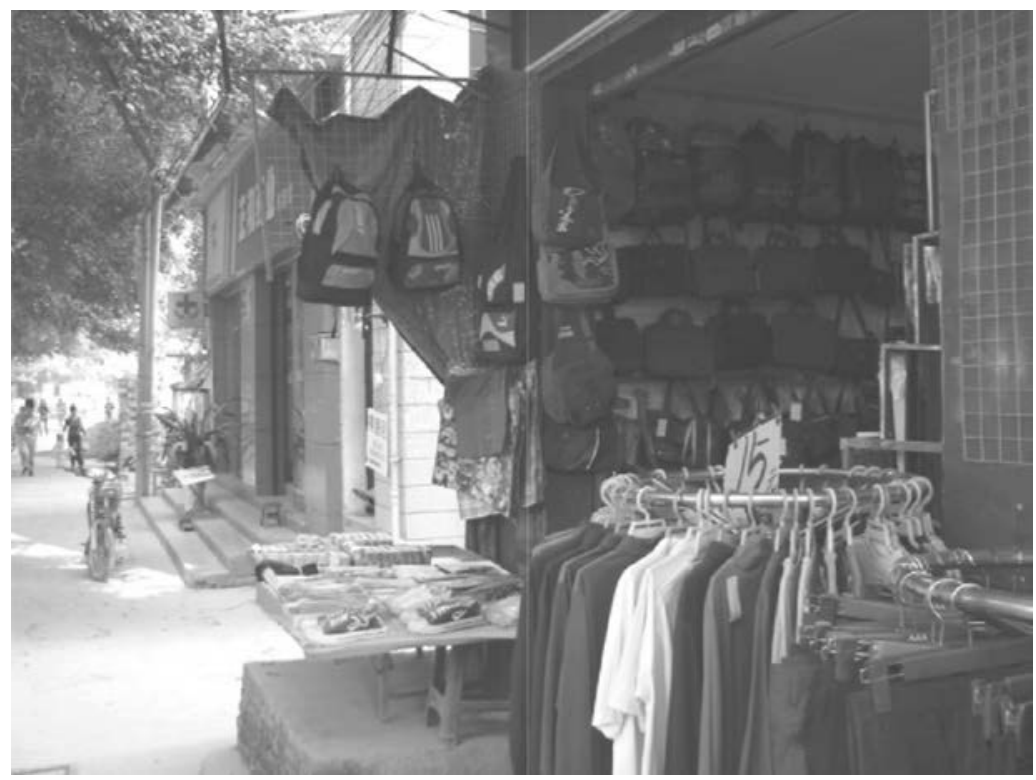

La vie étudiante au village consiste également en des aménagements tels des «places » où des tables sont disposées le soir pour la restauration. Les soirées étudiantes sont bruyantes et alcoolisées. Les temporalités de la journée dans le village sont en conséquence marquées et contrastées par les heures de travail des ouvriers sur les chantiers d'une part puis les découpages journaliers de la vie étudiante (aller et revenir des cours à l'université puis à des soirées à des heures tardives) d'autre part.

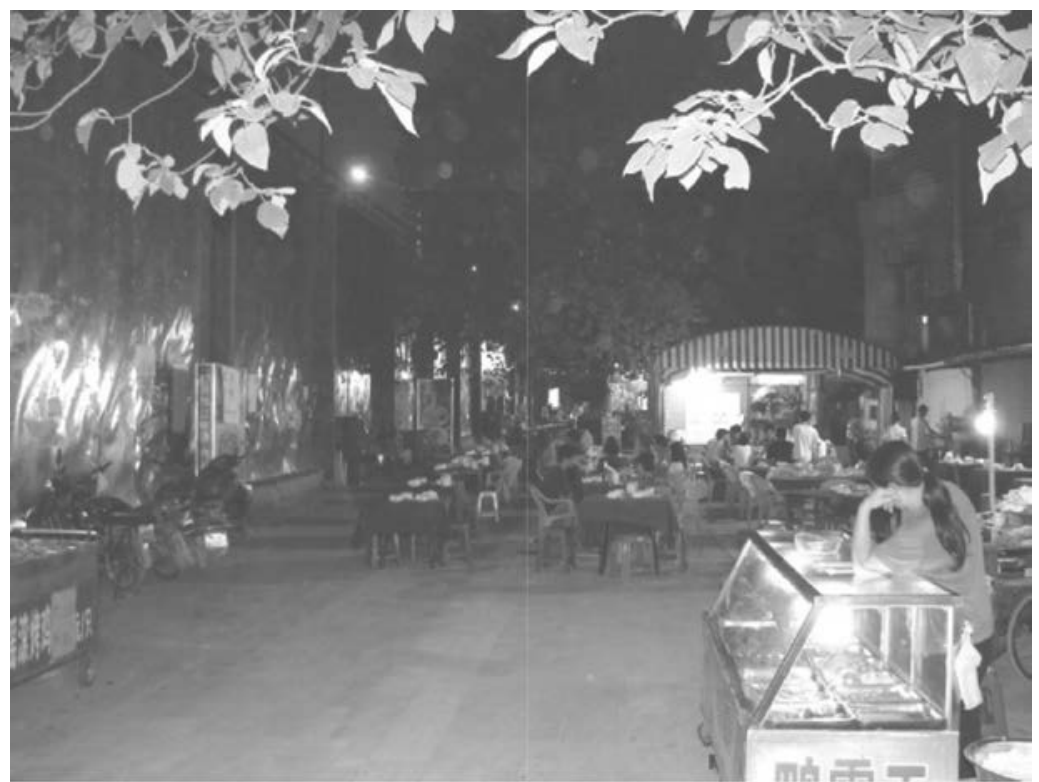




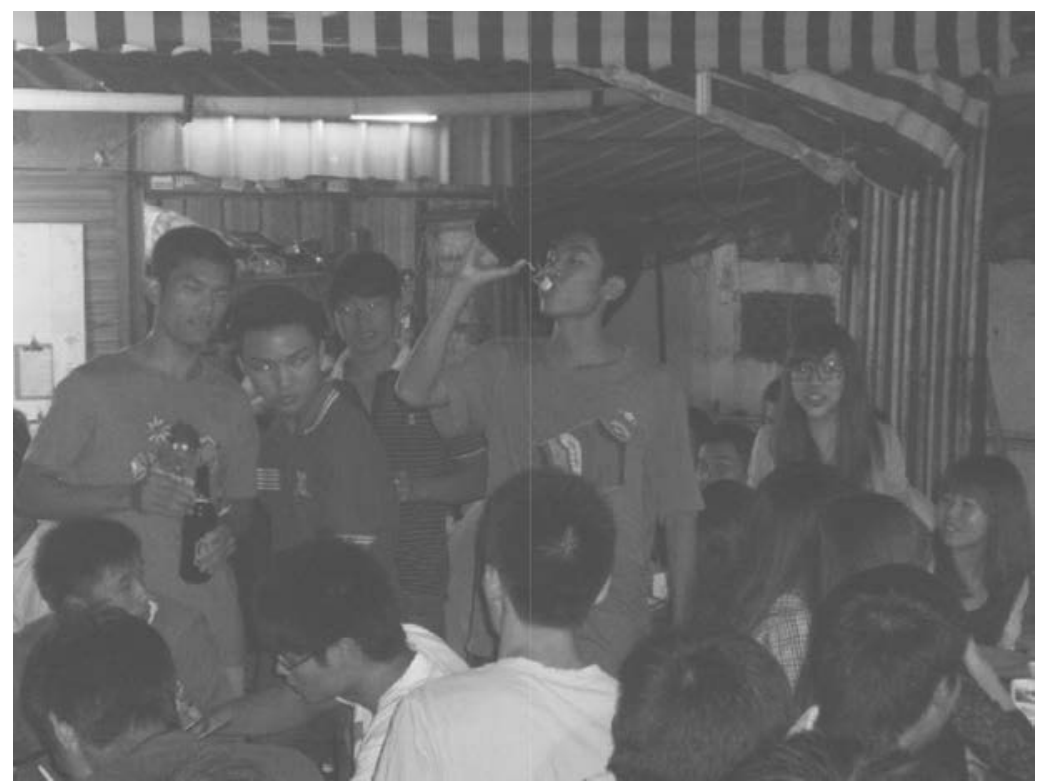

Photographie : auteurs, octobre 2011.

Dans le village de Nanting, une économie singulière et spatialisée est facilement identifiable et matérialisée. À proximité de l'université des beaux-arts de Canton, une vingtaine de petites échoppes de pinceaux, encadrement, dessins sont disséminées dans une des artères du village. Ces économies de niche, très spécialisées, sont liées aux conjonctures locales et aux effets d'aubaine - en matière d'opportunité d'emplois - perçus par les migrants.

E - Madame Lian X., gère un magasin d'encadrement avec son fils. Originaire du Hunan, est à la retraite (de son ancien métier, employée) et son mari est encore salarié dans sa province d'origine. Ce sont des liens de parenté explicites qui sont à l'origine de sa bifurcation professionnelle et sociale : «Je suis originaire de la province du Hunan. Je suis arrivée ici il y a un an pour ouvrir le magasin. Mon fils est en $4 \mathrm{e}$ année de licence en arts. Je suis venue ici pour le soutenir... je ne connaissais rien avant à ce métier, j'ai tout appris sur le tas. Je travaille principalement pour une galerie qui me commande régulièrement des cadres. » Pinceaux, cadres, encadrement, toiles, etc. sont vendus dans une multitude de petites échoppes à proximité de l'entrée du village de Nanting donnant directement sur l'université des Beaux-Arts. Si la rentabilité économique a été de mise selon Lian X., la concurrence est dorénavant élevée et les revenus ont baissé.

Les cas précités, issus de matériaux qualitatifs, témoignent explicitement des transformations sociales, démographiques et spatiales de l'île prises pour objet. Si les imposants édifices universitaires sont très visibles, il n'en demeure pas moins que les usages séculaires, les organisations sociales, les structures 
démographiques et les structures des secteurs d'emploi sont eux aussi largement bouleversés. La brusque et massive arrivée de la population étudiante et des services professionnels qui leur sont destinés a pour conséquence la cohabitation contrainte et dichotomique des nouveaux arrivants avec la population autochtone, constituée d'agriculteurs en majeure partie. Cette cohabitation se caractérise par des notions antagonistes : mobilité/immobilité ; allochtonie/autochtonie ; urbain/ rural ; ouverture culturelle/spécificité professionnelle ; nouvelles fonctions économiques/fonctions traditionnelles et coutumières, etc. Dès lors, ces contrastes sociaux et culturels visibles jusque dans les hexis corporelles [Bourdieu, 1979] peuvent-ils demeurer? Si oui, selon quelles logiques de négociations ?

\section{Conclusion : une ouverture vers la prise en compte des régulations politiques locales}

L'urbanisation rapide en Chine produit de multiples configurations spatiales, dont celle des «villages urbains ». Les contrastes entre les catégories de populations (villageois, migrants, étudiants) sont caractéristiques des propriétés spatiales des villages de l'île de Xiao Guwei. Aménagements rapides en moins d'une décennie, isolats spatiaux et sociaux et cohabitation de populations socialement très différentes au sein de mêmes lieux constituent les aspects typiques des cas étudiés. Mais, au-delà des interprétations relatives aux transformations sociales et spatiales de l'île et aux conséquences inhérentes, le processus analysé peut également être abordé sous l'angle de la sociologie politique, ouvrant dès lors de nouvelles questions et hypothèses de recherche.

Les entités administratives sont en effet doublées de cellules du parti communiste [Cabestan, 2014] « où se trouve le vrai pouvoir de décision » [Sanjuan, 2007]. En revanche, comme le droit d'utilisation du foncier est attribué aux villageois, se développe un ensemble de tensions, un « fort sentiment de défiance [...] de l'anxiété et un sentiment de privation » [Yingfang, 2009]. Pendant la phase de construction des complexes universitaires sur l'île de Xiao Guwei, le Bureau de pilotage du projet, qui est le principal organe où les décisions ont été prises, a multiplié les réunions thématiques, en accord avec les responsables « compétents » du parti communiste. Les membres de ce bureau ont ensuite été transférés à celui de la construction publique municipale de Canton. Cette multiplicité des échelons politiques et des organismes décisionnaires a eu pour effet de compliquer, pour les maires, la lecture des enjeux politiques. D'autant plus que, dans les villages observés, les conflits entre les différentes générations de migrants sont palpables. Ils génèrent une difficulté supplémentaire pour les maires qui profitent néanmoins de ces divisions, lors des négociations avec les autorités politiques hiérarchiques, pour essayer d'imposer leurs vues (expulsion des habitants logés illégalement, destruction des bâtiments construits pour loger les étudiants et les migrants).

Face aux enjeux du foncier sur l'île de Xiao Guwei, force est finalement de constater que les ressources individuelles et collectives (capital culturel, 
économique, réseaux de relations dans l'espace politique) ne permettent pas aux habitants et à leurs représentants élus de légitimer leurs visions de l'urbanisation des villages à l'égard du Bureau de la construction urbaine. Alors que des pétitions, des « fédérations d'habitants » ou encore des « communautés de quartier » émergent parfois, dans la ville de Pékin par exemple, à la faveur de propriétaires de logements urbains [Merle, 2014], le poids politique des habitants des villages de Beiting et Nanting n'a pas permis de maintenir un niveau de relations égal avec les représentants de l'État ou du Parti. Les maires (d'anciens petits agriculteurs) et les comités de villageois des deux villages pris pour objet, ne maîtrisant pas les codes politiques et ne disposant pas de ressources sociales et culturelles suffisamment efficaces ou efficientes, n'ont pas réussi à faire reconnaître leurs arguments auprès des autorités locales de ville de Canton et du Parti communiste.

\section{Bibliographie}

Arborio A.-M., Fournier P. [1999], L'Enquête et ses méthodes : l'observation directe, Paris, Nathan.

Aveline-Duchat N. [2013], «Les capitaux financiers à l'assaut de l'espace chinois », Perspectives chinoises, $\mathrm{n}^{\circ} 2$, p. 31-42.

Bourdieu P. [1979], La Distinction, critique sociale du jugement, Paris, Minuit.

CABESTAN J.-P. [2014], Le Système politique chinois. Un nouvel équilibre autoritaire, Paris, Presses de Sciences Po.

Chung H., Hunger J. [2013], « Le modèle d'urbanisation du Guangdong. Terres collectives et émergence d'une nouvelle classe moyenne dans les villages », Perspectives chinoises, $\mathrm{n}^{\circ} 3$, p. 35-44.

CONORD S. [2007], «Usages et fonctions de la photographie », Ethnologie française, vol. 37, $\mathrm{n}^{\mathrm{o}} 1$, p. 11-22.

DorIER-APPRILl E., JAGLIN S. [2002], « Introduction. Gestions urbaines en mutation : du modèle aux arrangements locaux », Autrepart, $\mathrm{n}^{\circ}$ 21, p. 5-15.

Gransow B. [2014], «Éditoriale », Perspectives chinoises, nº 2, p. 3-6.

Laurans V. [2006], «La nouvelle donne du logement à Shanghai », Transcontinentales, $n^{\circ} 3$, p. 31-46 : http://transcontinentales.revues.org/527 (consulté le 26 février 2015).

LE Quotidien de CANTON [2010], «Les fourmis urbaines emménagent à Beiting », 3 mai 2010 : http://gzdaily.dayoo.com/html/2010-05/03/content_951480.htm (consulté le 3 septembre 2019).

LI P. [2008], «Les "villages urbains" dans la Chine en mutation: le cas de Yangcheng à Canton », in Roulleau-Berger L., Guo Y., Li P., Liu S., La Nouvelle Sociologie chinoise, Paris, CNRS, p. 237-266.

Merle A., Youjun P. [2002], « Pékin entre modernisation et préservation », Perspectives chinoises, $\mathrm{n}^{\circ} 74$, p. 39-43.

Merle A. [2014], « Propriétaires de Pékin, unissez-vous ! La construction d'une mobilisation collective », Perspectives chinoises, $\mathrm{n}^{\circ}$ 2, p. 7-15.

PERSPECTIVES ChINOISES [2013], «L'urbanisation de la Chine rurale », $\mathrm{n}^{\circ} 3$. 
Piette A. [1996], Ethnographie de l'action. L'observation des détails, Paris, Métailié.

PiETte A. [2007], «Fondements épistémologiques de la photographie », Ethnologie française, vol. $37, \mathrm{n}^{\circ} 1$, p. 23-28.

Pinçon M., Pinçon-Charlot M. [1997], Voyage en grande bourgeoisie, Paris, Presses universitaires de France.

ROULLEAU-Berger L., Lu S. [2005], «Les travailleurs migrants à Shanghai. Inégalités, niches économiques et diversité des parcours d'accès à l'emploi », Perspectives chinoises, $\mathrm{n}^{\circ} 87$, p. 2-9.

Roulleau-Berger L. [2011], Désoccidentaliser la sociologie. L'Europe au miroir de la Chine, Paris, Éditions de L'Aube.

Roulleau-Berger L. [2012], Sociologies et cosmopolitisme méthodologique, Toulouse, Presses universitaires du Mirail.

RoulLEAU-BERGER L. [2013 a], « Métropolisation, frontières intérieures et "villes invisibles" en Chine », Espaces et sociétés, $\mathrm{n}^{\circ} 155$, p. 129-141.

Roulleau-Berger L. [2013 b], « Migrations internes, économies plurielles et bifurcations biographiques en Chine », Migrations Société : la revue du CIEMI, $\mathrm{n}^{\circ}$ 149, p. 77-89.

Sanjuan T. [2007], Atlas de la Chine contemporaine. Les mutations accélérées, Paris, Autrement.

YINGFANG C. [2009], « Légitimité, rationalité et stratégies politiques : les fondements du miracle urbain chinois », Terrains et travaux, $\mathrm{n}^{\circ} 16$, p. 97-136.

Zhong L. [2007], Guangzhou daxuecheng jianshe (la construction de Daxuecheng), Guanghzou, Éditions du peuple du Guangdong.

Zhu Y., Lin M., Lin L., Chen J. [2013], «Le phénomène de l'urbanisation in situ dans les districts ruraux en Chine. Le cas de la province du Fujian », Perspectives chinoises, $\mathrm{n}^{\circ} 3$, p. $45-55$. 